\title{
Patterns of recurrence after selective postoperative radiation therapy for patients with head and neck squamous cell carcinoma
}

\author{
Naoya Murakami ${ }^{*}$, Fumihiko Matsumoto ${ }^{2}$, Seiichi Yoshimoto ${ }^{2}$, Yoshinori Ito ${ }^{1}$, Taisuke Mori ${ }^{3}$, Takao Ueno ${ }^{4}$, \\ Keisuke Tuchida', Tairo Kashihara', Kazuma Kobayashi', Ken Harada', Mayuka Kitaguchi', Shuhei Sekii', \\ Rei Umezawa ${ }^{1}$, Kana Takahashi ${ }^{1}$, Koji Inaba ${ }^{1}$, Hiroshi Igaki ${ }^{1}$ and Jun Itami ${ }^{1}$
}

\begin{abstract}
Background: The radiation field for patients with postoperative head and neck squamous cell carcinoma is narrower in our institution than in Western countries to reduce late radiation related toxicities. This strategy is at a risk of loco-regional or distant metastasis. However, because patients are more closely checked than in Western countries by every 1 to 2 months intervals and it is supposed that regional recurrences are identified and salvage surgeries are performed more quickly. Therefore, it is considered that patient survival would not be compromised with this strategy. The aim of this study was to investigate the feasibility of this strategy retrospectively.

Methods: Patients who underwent neck dissection with close or positive margin, extra-capsular spread (ECS), multiple regional lymph node metastasis, pT4, with or without primary tumor resection were treated with postoperative radiation therapy. The volume of radiation field, especially the coverage of prophylactic regional lymph node area, was discussed among head and neck surgeons and radiation oncologists taking into account the clinical factors including patient's age, performance status, number of positive lymph nodes, size of metastatic lymph nodes, extension of primary tumor beyond the midline, and existence of ECS.

Results: Seventy-two patients were identified who were treated with postoperative radiation therapy for head and neck squamous cell carcinoma between November 2005 and December 2014. There were 20 patients with oropharynx, 19 with hypopharynx, 7 with larynx, 23 with oral cavity, and 3 with other sites. Thirty eight patients had their neck irradiated bilaterally and 34 unilaterally. Median follow-up period for patients without relapse was 20.7 months (5.1-100.7). Thirty two patients had disease relapse after treatment including 22 loco-regional recurrence and 14 distant metastases. Among 22 loco-regional recurrence, seven patients underwent salvage surgery and one of them was no relapse at the time of the analysis. Among patients without bilateral neck lymph node metastasis who were treated with unilateral neck irradiation, patients with oral cavity or recurrent disease had significantly lower DFS compared with those without (2-y DFS $41.7 \%$ vs $88.2 \%, p=0.017$ ).
\end{abstract}

Conclusions: In patients without bilateral neck lymph node involvement, the postoperative unilateral neck irradiation is a reasonable treatment strategy for patients with the exception of oral cavity or recurrent disease.

Keywords: Head and neck squamous cell carcinoma, Postoperative radiation therapy, Patterns of recurrence, Selective neck irradiation

\footnotetext{
* Correspondence: namuraka@ncc.go.jp

'Department of Radiation Oncology, National Cancer Center Hospital, 5-1-1

Tsukiji, Chuo-ku, Tokyo 104-0045, Japan

Full list of author information is available at the end of the article
} 


\section{Background}

According to statistics from Cancer Information Service in Japan, death from head and neck malignant tumors in Japan (malignant tumors arising from oral cavity, pharynx, and larynx) was 8142 in 2013 and this figure accounts for $2.2 \%$ of all the death from malignant tumors [1]. Although the percentage is decreasing, the smoking rate in 2013 was $32.2 \%$ in male and $8.2 \%$ in female and still many people smoke in our country [2].

In 1970's, Radiation Therapy Oncology Group (RTOG) 73-03 trial was carried out to compare preoperative with postoperative radiation therapy combined with surgical resection for patients with advanced operable squamous cell carcinoma of the supraglottic larynx or hypopharynx in the context of a phase III study [3]. Loco-regional control was significantly favorable for patients assigned to postoperative radiation therapy compared with those assigned to preoperative radiation therapy ( $65 \%$ vs $48 \%, p=0.04$ ), and the postoperative radiation therapy has been a standard of care for patients with advanced resectable head and neck squamous cell carcinoma (HNSCC). Nevertheless, development of distant metastasis was frequently observed in both arms and the addition of chemotherapy to surgery and adjuvant radiation therapy was considered as a next important issue. In the Intergroup study 0034 (or RTOG 85-03), a randomized clinical trial was conducted by cooperative groups which was consisted of RTOG, Southwest Oncology Group (SWOG), Eastern Cooperative Oncology Group (ECOG), Cancer and Leukemia Group B (CALGB), Northern California Oncology Group (NCOG), and Southwest Group (SEG), patients with advanced HNSCC were randomly assigned either to postoperative radiation alone or sequential three cycles of cis-platinum and 5-FU followed by postoperative radiotherapy [4]. While distant metastasis-free survival was significantly improved in sequential CT/RT arm (23\% vs $15 \%, p=0.02$ ), both locoregional relapse-free survival and overall survival did not differ between the two arms and the concomitant use of chemotherapy and radiation therapy was awaited. In 2004, the European Organization for Research and Treatment of Cancer (EORTC) and RTOG published simultaneously the results of two phase III trials (the EORTC 22931 and the RTOG 95-01) which compared concurrent postoperative chemoradiation using tri-weekly $100 \mathrm{mg} / \mathrm{m}^{2}$ of cisplatinum with postoperative radiotherapy alone $[5,6]$. There were slight differences in settings between these two phase III clinical trials. In the RTOG 95-01, the primary endpoint was the rates of local and regional control whereas, in the EORCT 22931 it was chosen to be the progression-free survival. The definition of the high-risk characteristics also differed between these two trials. In the RTOG 95-01, the following characteristics were defined as high-risk; histologic evidence of invasion of two or more regional lymph nodes, extra-capsular spread (ECS) of nodal disease, and microscopically involved mucosal margins of resection. On the other hand, in the EORTC 22931, the following characteristics were defined as high-risk; ECS, positive resection margins, perineural involvement, vascular tumor embolism, or tumors with involved lymph nodes at level IV or V from carcinomas arising in the oral cavity or oropharynx. While primary endpoint of these two phase III clinical trials were both met and overall survival benefit was demonstrated in the EORTC 22931 trial $(p=0.02)$, the RTOG 95-01 showed only a trend in the same direction in overall survival $(p=0.19)$. Bernier et al. conducted a comparative analysis using data pooled from the EORTC 22931 and the RTOG 95-01 to identify which patients require adjuvant concomitant chemoradiation following surgery and they concluded that microscopically involved resection margins and ECS of tumor from neck nodes were the most significant adverse factors for poor outcome [7]. Therefore, concurrent chemoradiation (cCRT) is a standard therapy for postoperative highrisk HNSCC patients.

Originally the radical neck dissection (RND) consists of removal of all the lymphatic as well as non-lymphatic structures from the mastoid process down to the clavicle except the carotid artery, brachial plexus, hypoglossal, lingual, vagus, and phrenic nerves $[8,9]$, which demands heavy burden to patients. Later on, selective neck dissection (SND) was introduced which preserved one or more lymph node levels [10] and the development of common terminology of discriminating neck levels which was well-known as the classification of American Head and Neck Society (AHNS) followed [11, 12]. However, the applicability of the concept of the selective nodal irradiation in postoperative setting is controversial [13-15]. Gregoire et al. [13] and Chao et al. [14] proposed the clinical target volume (CTV) guidelines for postoperative neck region, but the authors admitted the paucity of data on which one could create a specific guideline for postoperative CTV. According to the guideline of Chao et al., only patients with buccal T1-2 N0 and tonsil T1-2 N0 were allowed for hemi-neck postoperative radiation. After extensive neck irradiation patients usually are suffered from late radiation toxicities, in especially chemotherapy was administered concurrently with radiotherapy. In our institution radiation field for patients with postoperative head and neck squamous cell carcinoma is narrower than in Western countries to reduce late radiation related toxicities. This strategy is at a risk of loco-regional recurrence and/or distant metastasis. However, because patients are checked closely by every 1 to 2 months intervals 
and the salvage surgery would be performed immediately after the identification of the regional recurrences, therefore, it is considered that the patient's survival would not be compromised with this treatment strategy. This retrospective study was conducted to investigate the feasibility of this strategy.

\section{Methods}

All consecutive patients with HNSCC who underwent neck dissection and received postoperative radiation therapy were recruited for this study. In our institution, patients with HNSCC who underwent neck dissection with pathologic findings of close or positive resection margin, ECS, multiple regional lymph node metastasis, or pT4, with or without primary tumor resection were treated with postoperative radiation therapy. Surgical margin status was defined as follows; close margins were defined as $\leq 3 \mathrm{~mm}$ and positive margins defined as tumor touching an inked surface.

From April 2011 concurrent chemoradiation (cCRT) with tri-weekly CDDP $80 \mathrm{mg} / \mathrm{m}^{2}$, and from March 2013 Cetuximab-radiation according to the Bonner protocol [16] was introduced in our institution for patients with positive resection margin or ECS. Because there is no evidence supporting the superiority of Cetuximab-radiation over platinum-based cCRT in the management of advanced HNSCC, our first choice was CCRT. However, if patients did not have enough kidney function with favorable performance status, Cetuximab-radiation was chosen.

From June 2009 neoadjuvant chemotherapy (NAC) was started as chemoselection for patients with advanced HNSCC who required total laryngectomy or who expected severe postoperative pharyngeal dysfunction. If favorable response was achieved after two to three cycles of induction chemotherapy, subsequent cCRT was followed with or without neck dissection. If not, total laryngectomy or appropriate surgery was applied. NAC was also applied as induction chemotherapy for patients with far-advanced disease for whom it was impossible to separate metastatic lymph nodes from carotid artery which precludes radical operation or patients with N2c and/or lower neck metastasis who's possibility of developing distant metastasis soon after surgery was expected to be very high. Agents used for NAC was either the combination of CDDP and 5FU or CDDP, 5FU, and docetaxel.

The extent of prophylactic neck resection was determined by the status of primary lesion. If primary lesion extended midline, prophylactic contralateral neck dissection was applied. Otherwise, unilateral prophylactic neck dissection was performed.

Patients with distant metastasis, treated for palliative intention, or for salvage intention after recurrence without surgical resection were excluded from this study.

\section{Radiotherapy}

Radiotherapy was prescribed in 2-Gy fractions with 4 or 6-MV photons in either three-dimensional conformal radiotherapy (3DCRT) or intensity-modulated radiotherapy (IMRT). From September 2008 a simultaneous integrated boost intensity-modulated radiotherapy (SIB-IMRT) using sliding window technique or volumetric modulated arc therapy (VMAT) by dynamic MLC system (Varian Medical Systems, Palo Alto, CA) was introduced in our institution, in case of the CTV contained large volume of major salivary gland, oral cavity, larynx, or pharynx. Our IMRT procedure for head and neck cancer patients is described in a previous report [17]. Patients were immobilized from head to shoulders with thermoplastic masks in the supine position. Target volumes were defined as follows: no gross tumor volume (GTV) was defined except patients without primary lesion resection because all gross tumor was resected during operation. The high-risk CTV 60-66 Gy $\left(\mathrm{CTV}_{60-66 G y}\right)$ was defined as areas considered as high risk for having microscopic disease such as positive surgical margin or metastatic lymph node with ECS based on preclinical imaging, preoperative physical exam/endoscopy, operative findings, and final pathologic findings. The intermediate-risk CTV (CTV 44Gy $_{\text {for }}$ 3DCRT and $\mathrm{CTV}_{54 \mathrm{~Gy}}$ for IMRT) included the cervical lymphatic pathways which are considered to be at risk for having potential microscopic disease. The extent of the $\mathrm{CTV}_{44 \mathrm{~Gy}}$ or the CTV $\mathrm{CHGy}_{\text {Gy }}$ was discussed among head and neck surgeons and radiation oncologists taking into account of clinical factors including patient's age, performance status, number and distribution of positive lymph nodes, size of metastatic lymph nodes, extension of primary tumor beyond the midline, pathological resection status, and existence of ECS. For example, patients with ipsilateral multiple neck lymph node metastases with large nodes more than $3 \mathrm{~cm}$ in diameter and/or multiple ECSs generally received prophylactic contralateral neck irradiation. However, if patients were elderly and fragile, prophylactic contralateral neck irradiation was often omitted. If patients received total laryngectomy, the risk of acquiring aspiration pneumonia was reduced, therefore, threshold of providing prophylactic contralateral neck irradiation would be lowered.

After completion of radiotherapy, patients were closely followed by every 1 to 2 months for the initial 2 years, every 3 to 4 months for years $3-5$, and once or twice a year thereafter. When surgically resectable recurrent lymph nodes were identified in the regional neck area without distant metastasis during the follow-up visits and patients had favorable performance status, a salvage surgery would be performed immediately after the identification. 


\section{Statistics}

Survival curves were estimated by using the KaplanMeier method and the differences were assessed by the log-rank test. The relationships between clinical and treatment variables and disease-free survival (DFS) were analyzed by the univariate analysis. Student's unpaired $t$ test was used to compare the continuous variables and Pearson's chi-square test to compare the categorical variables. A $\mathrm{P}$ value of $\leq 0.05$ was considered statistically significant. Factors with $p$ value $\leq 0.05$ were further analyzed in the multivariate analysis by the Cox regression analysis. This analysis was intended to find out a most appropriate population suitable for postoperative unilateral-RT, so the promising factors were combined and analyzed by the multivariate analysis. However, to eliminate the statistics confounding, factors were used in the multivariate analysis only once. The Statistical analysis was performed using SPSS Statistics (version 18.0; SPSS, Inc., Chicago, IL).

This retrospective study was approved by the institutional ethical review board of the National Cancer Center Hospital. This retrospective study was performed in accordance with the ethical standards laid down in the 1964 Declaration of Helsinki and its later amendments.

\section{Results}

From November 2005 and December 2014, 72 patients were identified who underwent neck dissection and postoperative radiation therapy for HNSCC. Pretreatment patient and tumor characteristics are summarized in Table 1. There were 20 patients with oropharynx, 19 with hypopharynx, 7 with larynx, 23 with oral cavity, and 3 with other sites. Because HPV infectious status has been routinely assessed since 2011, only 6 out of 20 patients of oropharyngeal cancer patients were assessed for HPV and 5 of them (83.3\%) were positive for HPV. Two patient were stage III, 48 IVA, 3 IVB, and 19 after salvage surgery for recurrent disease. In recurrent cases, they were classified into either stage rIII or rIVA. Thirty eight patients had their neck irradiated bilaterally and 34 unilaterally. There was no difference between bilateral and unilateral neck irradiated cohorts except number of lymph node metastases. Statistically more patients had more than two lymph node metastases in bilateral neck cohort than unilateral neck cohort $(p=0.031)$, suggesting that more advanced patients were treated by bilateral neck irradiation. There was one patient with N2c who received unilateral neck irradiation. Because this 71 years old patient had past history of subtotal esophagectomy, left upper lobe segmentectomy, and major depression, and his contralateral side of neck lymph node was without ECS, therefore, it was decided that contralateral neck should be omitted for prophylactic irradiation to reduce toxicity. Pathological characteristics and treatment details are summarized in Table 2. Eight patients received NAC before surgery. Seven patients received the combination of CDDP and 5FU and one the combination of docetaxcel, CDDP, and 5FU. Three patients had their primary lesion treated by brachytherapy and nine patients by external beam radiation therapy. The others had their primary lesion as well as regional neck area surgically resected. Among 60 patients whose primary tumor had surgically resected, 38 patients had their primary site irradiated mainly because of positive/close resection margin or T4 disease. Statistically fewer patients were treated by 3DCRT in unilateral neck cohort than in bilateral neck cohort $(p=0.031)$.

Median follow-up period for patients without failure was 20.7 months (range, 5.1-100.7 months). 2-year Overall survival (OS), DFS, and Loco-regional control (LRC) were 66.0, 53.4, and 66.0\%, respectively (Fig. 1). Pattern of the first relapse is summarized in Table 3. Thirty two patients had disease relapse after treatment including 22 loco-regional recurrence and 14 distant metastases. Significantly more patients with failure were identified in bilateral neck cohort $(p=0.015)$. Ten patients developed in-field recurrence. Nodal failure was found within the high-risk CTV in seven patients and within the intermediate-risk CTV in three patients. Twelve patients were identified with extra-field locoregional failure: three recurrences were found in ipsilateral retropharyngeal lymph node, one in ipsilateral level Ib and IV simultaneously, two in ipsilateral level V, two in tumor bed region, one in the nasopharynx, two in neck skin, and one in non-irradiated contralateral neck region. The most frequently affected site as a distant metastasis was lung, following bone and mediastinal lymph node. Among 22 loco-regional recurrence, seven patients underwent salvage surgery although only one patient remains without relapse at the time of the analysis. Potential factors influencing DFS were summarized in Table 4. In the univariate analysis, IMRT was found out to be factors for favorable DFS. On the other hand, more than two lymph node metastasis, oral cavity primary or recurrent disease, T4 or recurrent disease, and oral cavity primary or recurrent disease were identified to be factors for unfavorable DFS. The aim of this study was to find a group of patients who could be safely applied unilateral neck irradiation and generally it is natural to irradiate bilateral neck for patients with bilateral neck lymph node involvement. Consequently, all 10 patients with bilateral neck lymph node metastasis were excluded and uni- and multivariate analysis was performed (Table 5). In the multivariate analysis, it was found that inferior DFS correlated with oral cavity or recurrent disease (Hazard Ratio 1.696; 95 \% confidence interval 1.29_ 1.87, Fig. 2). Among patients with unilateral lymph node metastasis treated with unilateral neck irradiation, oral cavity or recurrent disease were adverse factors for DFS 
Table 1 Patient and tumor characteristics

\begin{tabular}{|c|c|c|c|}
\hline & $\begin{array}{l}\text { Bilateral RT } \\
(n=38)\end{array}$ & $\begin{array}{l}\text { Unilateral RT } \\
(n=34)\end{array}$ & $p$ value \\
\hline \multicolumn{4}{|l|}{ Primary site } \\
\hline Oral cavity & 10 & 13 & 0.556 \\
\hline Oropharynx & 12 & 8 & \\
\hline Hypopharynx & 10 & 9 & \\
\hline Larynx & 5 & 2 & \\
\hline Others & 1 & 2 & \\
\hline \multicolumn{4}{|l|}{ T-classification } \\
\hline $\mathrm{T} 1$ & 4 & 4 & 0.673 \\
\hline $\mathrm{T} 2$ & 13 & 11 & \\
\hline T3 & 5 & 4 & \\
\hline T4 & 8 & 4 & \\
\hline $\operatorname{Rec}$ & 8 & 11 & \\
\hline rTO & 6 & 8 & \\
\hline $\mathrm{rT1}$ & 0 & 0 & \\
\hline rT2 & 2 & 2 & \\
\hline rT3 & 0 & 0 & \\
\hline rT4 & 0 & 1 & \\
\hline \multicolumn{4}{|l|}{ N-classification } \\
\hline No & 0 & 2 & 0.124 \\
\hline $\mathrm{N} 1$ & 0 & 0 & \\
\hline $\mathrm{N} 2 \mathrm{a}$ & 3 & 2 & \\
\hline $\mathrm{N} 2 \mathrm{~b}$ & 18 & 17 & \\
\hline $\mathrm{N} 2 \mathrm{C}$ & 8 & 1 & \\
\hline N3 & 1 & 1 & \\
\hline $\operatorname{Rec}$ & 8 & 11 & \\
\hline $\mathrm{rN1}$ & 1 & 1 & \\
\hline rN2 & 7 & 10 & \\
\hline rN3 & 0 & 0 & \\
\hline \multicolumn{4}{|l|}{ Stage } \\
\hline III & 1 & 1 & 0.637 \\
\hline IVA & 28 & 20 & \\
\hline IVB & 1 & 2 & \\
\hline $\operatorname{Rec}$ & 8 & 11 & \\
\hline rlll & 1 & 1 & \\
\hline rIVA & 7 & 10 & \\
\hline \multicolumn{4}{|c|}{ Bilateral neck LN metastasis } \\
\hline Yes & 9 & 1 & $0.011^{*}$ \\
\hline No & 29 & 33 & \\
\hline \multicolumn{4}{|l|}{ Necrosis in LN } \\
\hline Yes & 21 & 21 & 0.401 \\
\hline No & 14 & 9 & \\
\hline Unknown & 3 & 4 & \\
\hline
\end{tabular}

Table 1 Patient and tumor characteristics (Continued)

\begin{tabular}{|c|c|c|c|}
\hline \multicolumn{4}{|c|}{ Maximum diameter of LN (cm) } \\
\hline Median & 2.5 & 2.7 & 0.783 \\
\hline Range & $0.4-7.5$ & $0.8-4.1$ & \\
\hline \multicolumn{4}{|c|}{ Number of $L N$ metastasis } \\
\hline $0-1$ & 6 & 13 & $0.031^{*}$ \\
\hline$\geq 2$ & 32 & 21 & \\
\hline \multicolumn{4}{|l|}{ Sex } \\
\hline Male & 32 & 26 & 0.217 \\
\hline Female & 6 & 8 & \\
\hline \multicolumn{4}{|l|}{ Age } \\
\hline Median & 63 & 63 & 0.270 \\
\hline Range & $38-80$ & $34-84$ & \\
\hline
\end{tabular}

(2-y DFS $41.7 \%$ vs $88.2 \%, p=0.017$, Fig. 3). On the other hand, among patients who had unilateral lymph node metastasis treated with bilateral neck irradiation, no statistically significant difference was found but a tendency towards inferior DFS for patients with oral cavity or recurrent disease compared to that of those without (2-y DFS $22.2 \%$ vs $60.9 \%, p=0.056)$. Out of 40 relapsefree patients, one patient was para-enteral nutrition dependent, two patients developed hypothyroidism requiring the hormone replacement treatment, one patient developed ulcer at tonsil which resolved conservatively, one patient developed severe dry mouth which always required water to moisten the mouth, two patients developed metachronous malignancy in head and neck region, and two patients died of intercurrent disease (one died of subarachnoid hemorrhage and one liver cirrhosis). The one who remained para-enteral nutrition dependent was treated by bilateral neck irradiation.

\section{Discussion}

The relapse rate was significantly higher in bilateral neck cohort compared with unilateral neck cohort although larger volume being irradiated (Table $3, p=0.015$ ). The possible explanation of this unfavorable results in bilateral neck cohort was that statistically more patients with two or more lymph node metastases were treated by bilateral radiation therapy (Table 1 ).

Eisbruch et al. reported that there existed dose-volume relationship between the pharyngeal constrictors, the glottic, and supraglottic larynx and late radiation complications such as dysphagia and aspiration [18]. Because our study was only a retrospective study and it was not possible to collect reliable data concerning late neck toxicities. Therefore, it was not possible to show inferior quality of life for patients who were treated by bilateral neck irradiation compared with those who were treated 
Table 2 Pathological characteristics and treatment details

\begin{tabular}{|c|c|}
\hline \multicolumn{2}{|l|}{ NAC } \\
\hline Yes & 8 \\
\hline No & 64 \\
\hline \multicolumn{2}{|l|}{ Bi-lateral ND } \\
\hline Yes & 26 \\
\hline No & 46 \\
\hline \multicolumn{2}{|l|}{ Treatment for primary lesion } \\
\hline Surgery & 60 \\
\hline EBRT & 9 \\
\hline BT & 3 \\
\hline \multicolumn{2}{|l|}{ Degree of differentiation } \\
\hline Poorly differentiated & 20 \\
\hline Moderately differentiated & 23 \\
\hline Well differentiated & 15 \\
\hline Unknown & 14 \\
\hline \multicolumn{2}{|l|}{ Extracapsular spread } \\
\hline Yes & 46 \\
\hline No & 10 \\
\hline Unknown & 16 \\
\hline \multicolumn{2}{|l|}{ Positive/close margin } \\
\hline Yes & 40 \\
\hline No & 32 \\
\hline \multicolumn{2}{|l|}{ Concurrent systemic therapy } \\
\hline CDDP & 17 \\
\hline Cetuximab & 2 \\
\hline TS-1 & 2 \\
\hline None & 51 \\
\hline \multicolumn{2}{|l|}{ RT total dose (Gy) } \\
\hline Median & 66 \\
\hline Range & $60-74$ \\
\hline \multicolumn{2}{|l|}{ Radiation technique } \\
\hline 3DCRT & 14 \\
\hline IMRT & 58 \\
\hline
\end{tabular}

$R T$ radiation therapy, $N A C$ neoadjuvant chemotherapy, ND neck dissection, $E B R T$ external beam radiation therapy

$B T$ brachytherapy, $L N$ lymph node, $3 D C R T$ three-dimensional conformal radiation therapy, IMRT intensity modulated radiation therapy

by unilateral neck irradiation. However, it is common in daily practice to see patients with bilateral neck irradiation who are suffered from neck stiffness or shoulder discomfort which not merely worsen patient's quality of life but also hinders early detection of neck lymph node recurrence or make it difficult to perform possible salvage surgery. Therefore, if it is feasible, to reduce acute and late complications related to irradiation it is obviously desirable to irradiate as smaller volume as possible. There were only two patients with NO in this study
(Table 1), but large T classification T3 with positive margin and T4. Hence, if the guideline for postoperative radiation therapy created by Chao et al. [14] would have been applied to our patients, theoretically all the patients should have been treated by bilateral neck irradiation. This patient with T3N0 and positive margin eventually developed local and regional recurrence. Because there were only two patients with postoperative N0 in our study, it is difficult to make any recommendations of postoperative radiation therapy for postoperative NO patients. However, because one among the two NO patients developed regional recurrence, prophylactic postoperative radiation seems to be also important for postoperative N0 patients with high risk pathological features.

It was observed in this study that seven out of $22 \mathrm{pa}-$ tients with loco-regional recurrence could undergo salvage surgery and only one of them eventually achieved no relapse at the time of the analysis. Therefore, salvage surgery had only minor impact on patient's overall survival. This finding was in line with a recent randomized phase III trial comparing elective neck dissection or watchful wait with close follow up for early-stage oral cancer. The latter strategy was significantly inferior in overall survival rate despite the protocol mandated close follow-up for neck examination [19]. In this study, patients who developed nodal relapse presented with a more advanced nodal stage and a higher prevalence of ECS than initial presentation, which possibly made it more difficult to control disease by salvage interventions. Accordingly, finding patients who are unlikely to develop loco-regional recurrence after unilateral neck irradiation seems to be a better treatment strategy.

HNSCC with a positive human papilloma virus (HPV) has been recently reported to be radiosensitive $[20,21]$. $\mathrm{Ki}-67$ and p53 were also reported to be prognostic markers for HNSCC postoperative radiotherapy [22]. The prognostic impact of these markers on survival for patients with HNSCC who were treated with postoperative radiation therapy could not be assessed because only part of patients were examined for p16, Ki-67, and p53 status in this study. Similarly, although ECS of lymph node is a well-known major adverse pathological factor among patients of HNSCC [5-7] and description concerning ECS of lymph node has been found since 2005, it was only from 2011 that documentation about ECS of lymph node has been made without exception. Therefore, there were as many as 16 missing data and the prognostic impact of ECS of lymph node could not be found in our study. Resection margin status is also a well-known major adverse pathological factor [5-7]. However, in our study adverse prognostic feature of resection margin status could not be shown presumably because patients with positive/close margin received postoperative radiation therapy appropriately. On the 


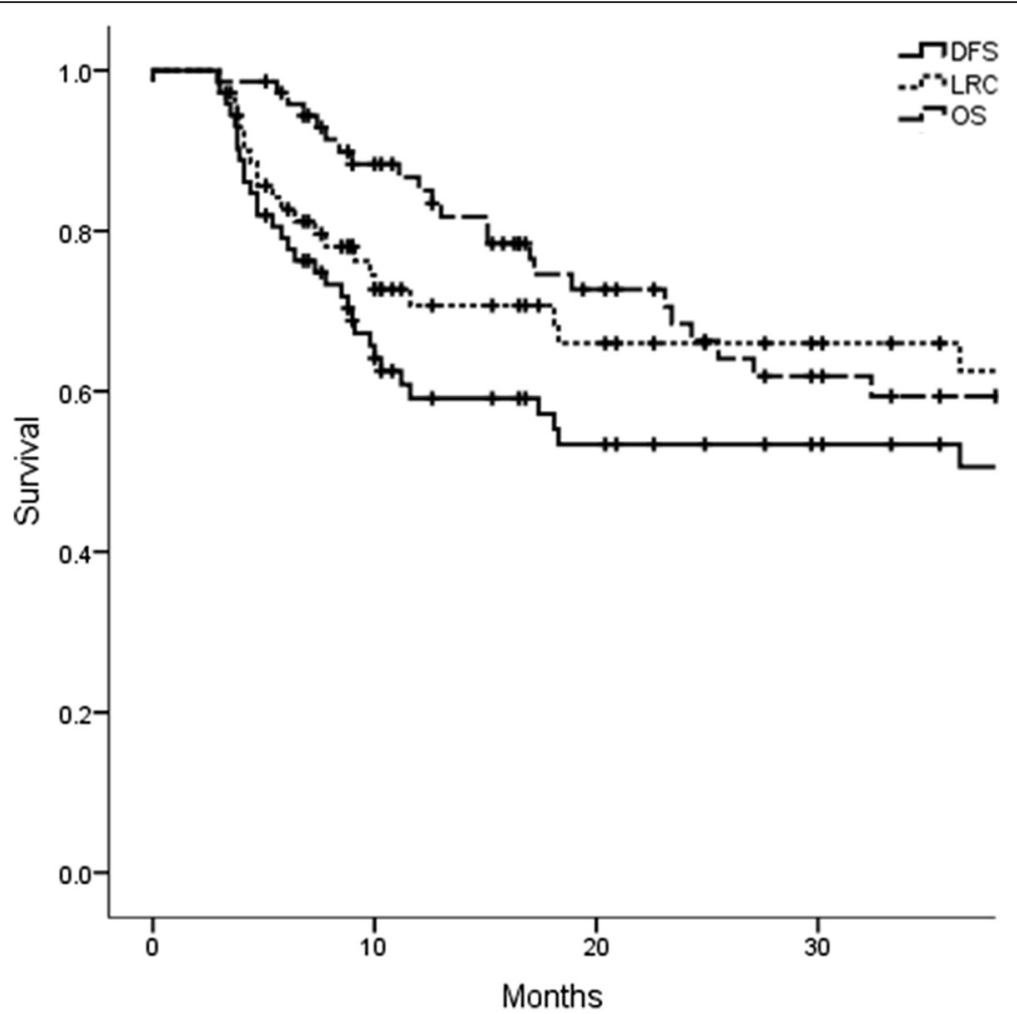

Fig. 1 Kaplan-Meyer curves of overall survival (OS), disease-free survival (DFS), and loco-regional control (LRC)

Table 3 Pattern of first failures

\begin{tabular}{|c|c|c|c|}
\hline & $\begin{array}{l}\text { Bilateral RT } \\
(n=38)\end{array}$ & $\begin{array}{l}\text { Unilateral RT } \\
(n=34)\end{array}$ & $p$ value \\
\hline \multicolumn{4}{|c|}{ Any failure } \\
\hline Yes & 22 & 10 & \multirow[t]{2}{*}{$0.015^{*}$} \\
\hline No & 16 & 24 & \\
\hline \multicolumn{4}{|c|}{ Loco-regional failure } \\
\hline Yes & 15 & 7 & \multirow[t]{2}{*}{0.082} \\
\hline No & 23 & 27 & \\
\hline \multicolumn{4}{|c|}{ In-field failure } \\
\hline Yes & 8 & 2 & \multirow[t]{2}{*}{0.062} \\
\hline No & 30 & 32 & \\
\hline \multicolumn{4}{|c|}{ Extra-field loco-regional failure } \\
\hline Yes & 7 & 5 & \multirow[t]{2}{*}{0.572} \\
\hline No & 31 & 29 & \\
\hline \multicolumn{4}{|c|}{ Distant failure } \\
\hline Yes & 10 & 4 & \multirow[t]{2}{*}{0.119} \\
\hline No & 28 & 30 & \\
\hline
\end{tabular}

$R T$ radiation therapy. ${ }^{*} \mathrm{~A} P$ value of $\leq 0.05$ was considered statistically significant
Table 4 Potential predictors influencing DFS

\begin{tabular}{llll}
\hline & \multicolumn{2}{l}{ 2-y DFS (\%) } & DFS \\
\cline { 2 - 3 } & yes & no & \\
\hline OC & 38.9 & 59.8 & 0.067 \\
Necrosis in LN & 63.8 & 49.1 & 0.245 \\
Bi-lateral ND & 50.3 & 54.7 & 0.654 \\
NAC & 87.5 & 48.6 & 0.076 \\
Rec & 30.6 & 59.6 & 0.062 \\
T4 & 37.5 & 56.2 & 0.21 \\
LN+ $\geq 2$ LN+ $\geq 2$ & 44.9 & 76.5 & $0.019^{*}$ \\
Extracapsular extention & 50.7 & 62.5 & 0.177 \\
Positive/close margin & 48.5 & 57.2 & 0.82 \\
Bilateral RT & 42 & 68.4 & 0.057 \\
IMRT & 69.5 & 35.7 & $0.047^{*}$ \\
Systemic therapy & 38.7 & 57.1 & 0.449 \\
OC or rec & 33.9 & 66.4 & $0.013^{*}$ \\
T4 or rec & 36.7 & 65.7 & $0.012^{*}$ \\
OC or T4 & 37.5 & 65.1 & $0.012^{*}$ \\
OC or T4 or rec & 37.9 & 72.4 & $0.006^{*}$ \\
\hline DFS disease free survit & & &
\end{tabular}

DFS disease free survival

$O C$ oral cavity, $L N$ lymph node, $N D$ neck dissection, Rec recurrence. *A $P$ value of $\leq 0.05$ was considered statistically significant 
Table 5 Potential predictors influencing DFS for patients excluding bilateral neck lymph node metastasis

\begin{tabular}{|c|c|c|c|c|c|c|}
\hline & $2-y D F$ & & DFS & & & \\
\hline & yes & no & $p$ value in uni. & $p$ value in multi. & $H R$ & $95 \% \mathrm{Cl}$ \\
\hline OC & 36.9 & 64.4 & $0.034^{*}$ & & & \\
\hline Necrosis in LN & 64.9 & 48.4 & 0.423 & & & \\
\hline Bi-lateral ND & 53.0 & 55.9 & 0.998 & & & \\
\hline NAC & 87.5 & 48.6 & 0.076 & & & \\
\hline $\operatorname{Rec}$ & 30.6 & 64.2 & $0.029^{*}$ & & & \\
\hline T4 & 46.7 & 56.6 & 0.398 & & & \\
\hline $\mathrm{LN}+\geq 2 \mathrm{LN}+\geq 2$ & 45.3 & 76.5 & $0.032^{*}$ & 0.062 & & \\
\hline Extracapsular extention & 52.2 & 57.1 & 0.442 & & & \\
\hline Positive/close margin & 56.1 & 54.2 & 0.824 & & & \\
\hline Bilateral RT & 44.4 & 67.3 & 0.146 & & & \\
\hline IMRT & 60.8 & 33.3 & $0.050^{*}$ & 0.162 & & \\
\hline Systemic therapy & 43.3 & 56.5 & 0.619 & & & \\
\hline OC or rec & 31.9 & 74.0 & $0.004^{*}$ & 0.006 & 1.696 & $1.29-1.87$ \\
\hline
\end{tabular}

DFS disease free survival, uni. univariate analysis, multi. multivariate analysis, $H R$ hazard ration, $C l$ confidence interval, $O C$ oral cavity, $L N$ lymph node, $N D$ neck dissection, $R e c$ recurrence. ${ }^{*} A P$ value of $\leq 0.05$ was considered statistically significant

other hand, multivariate analysis in this study revealed that patients with oral cavity or recurrent disease were significantly worse DFS compared with those without and its disease-free survival disadvantage was $69.6 \%$ (Table 5, Fig. 2). Radiation resistance of tumors from oral cavity [23] has been reported previously, therefore, current findings were clinically comprehensible. Among patients without bilateral neck node metastasis and treated with unilateral neck irradiation, patients with oral cavity or recurrent disease had significantly inferior DFS compared with those without (2-y DFS $41.7 \%$ vs $88.2 \%, p=0.017$, Fig. 3). Therefore, in patients without bilateral neck lymph

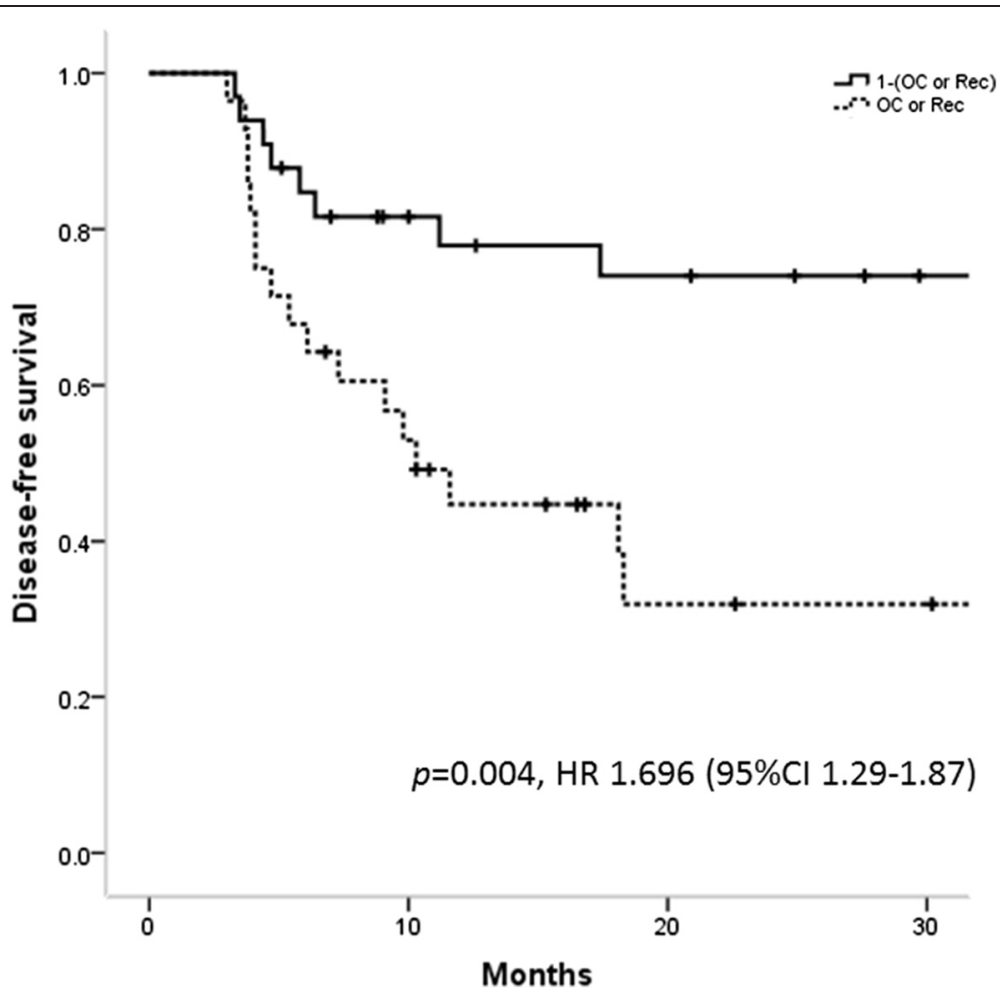

Fig. 2 Disease-free survival (DFS) stratified by the group of patients with oral cavity or recurrent disease or those without 


\section{Patients treated by uni-lateral RT $(n=33)$}

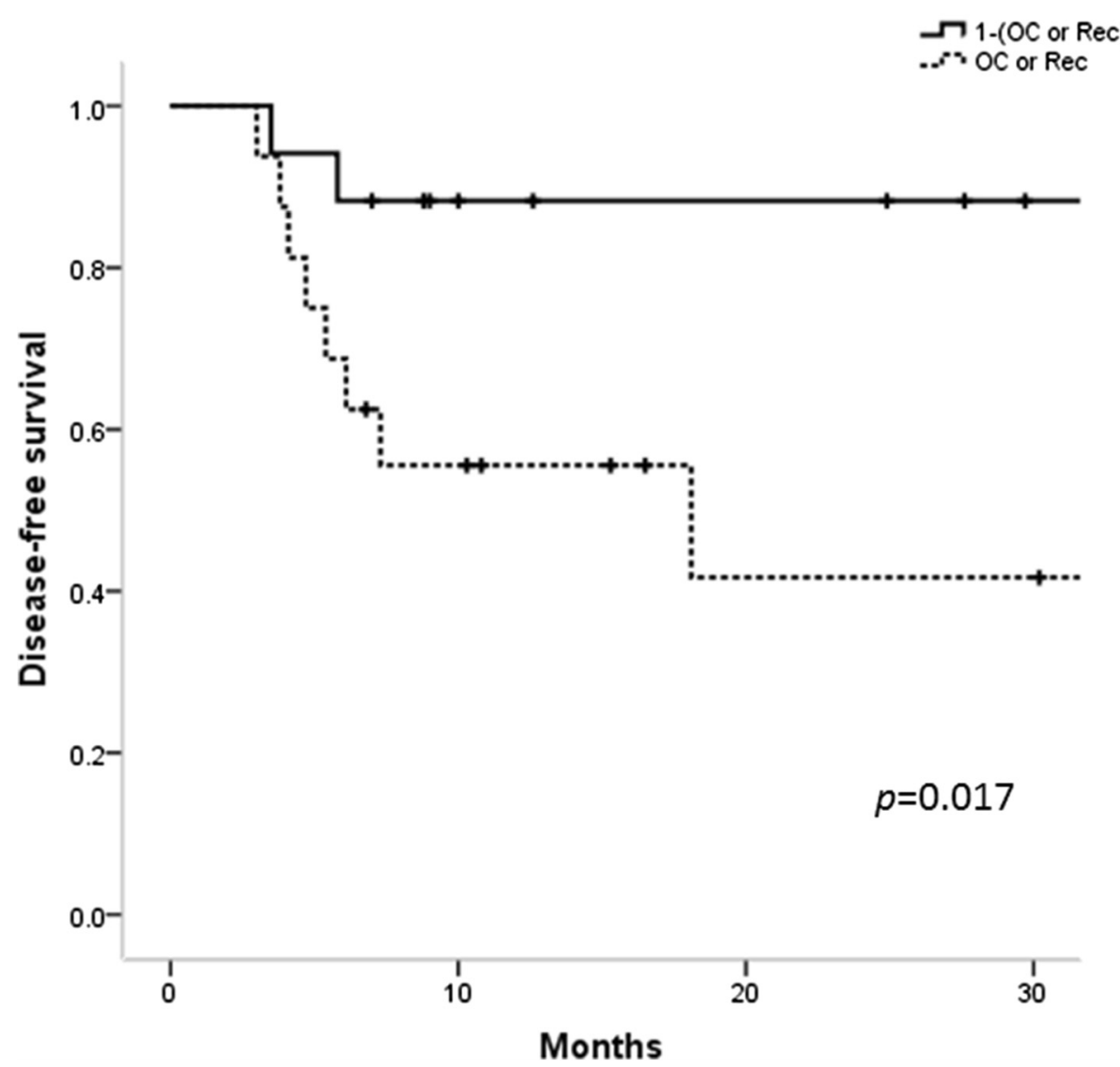

Fig. 3 Disease-free survival (DFS) for patients treated by unilateral neck cohort. Survival curves were stratified by the group of patients with oral cavity or recurrent disease or those without

node involvement, the postoperative unilateral neck irradiation is a reasonable treatment strategy for patients with the exception of oral cavity or recurrent disease.

On the contrary, for patients with oral cavity origin or recurrent disease, bilateral neck irradiation did not seem to be a promising solution. If bilateral neck irradiation was a favorable solution for patients with oral cavity or recurrent disease, DFS should have been superior for patients with bilateral neck irradiation to those with unilateral neck irradiation. However, among patients with oral cavity or recurrent disease, 2-y DFS for patients with bilateral and unilateral neck irradiation were $22.2 \%$ and $41.7 \%$, respectively $(p=0.412)$. Thus, different approaches should be made to improve the clinical results for patients with oral cavity or recurrent disease. In this study, the most frequent site of regional recurrence was the high-risk CTV (70 \%), similar results showed with Carrillo et al. [15]. Out of field regional recurrence was observed more frequently in ipsilateral neck than contralateral neck whereas only one patient developed contralateral-neck failure. Because concurrent CDDP administration was only started since 2008 in our institution, the majority patients did not received cCRT in this analysis, which could be a possible explanation for many loco-regional recurrences. Therefore, dose escalation for the high-risk CTV or application of cCRT or widening the intermediate-risk CTV in ipsilateral neck region to submandibular or posterior neck would possibly decrease the rate of loco-regional recurrence in the future.

There were several limitations in this study. Treatment strategy and radiation field was not uniformed according to several patient's clinical backgrounds. For example, treatment plans were heterogeneous including bioradiation, chemoradiation, or radiation alone. And the chemotherapy agents used were not unified. Also, this study was a retrospective study consisted of a small number of patients from single institution. In spite of these drawbacks, several insights were derived from this analysis which would possible improve treatment in the future. 


\section{Conclusions}

In patients without bilateral neck lymph node involvement, the postoperative unilateral neck irradiation is a reasonable treatment strategy for patients with the exception of oral cavity or recurrent disease.

\begin{abstract}
Abbreviations
3DCRT: three-dimensional conformal radiotherapy; AHNS: American Head and Neck Society; CALGB: Cancer and Leukemia Group B; CCRT: concurrent chemoradiation; CTV: clinical target volume; DFS: disease-free survival; ECOG: Eastern Cooperative Oncology Group; ECS: extra-capsular spread; EORTC: European Organization for Research and Treatment of Cancer; GTV: gross tumor volume; HNSCC: head and neck squamous cell carcinoma; HPV: human papilloma virus; IMRT: intensity-modulated radiotherapy; LRC: loco-regional control; NAC: neoadjuvant chemotherapy; NCOG: Northern California Oncology Group; OS: overall survival; RND: radical neck dissection; RTOG: Radiation Therapy Oncology Group; SEG: Southwest Group; SIBIMRT: simultaneous integrated boost intensity-modulated radiotherapy; SND: selective neck dissection; SWOG: Southwest Oncology Group; VMAT: volumetric modulated arc therapy.
\end{abstract}

\section{Competing interests}

The authors declare that they have no competing interests.

\section{Authors' contributions}

NM, FM, and SY have made substantial contributions to conception and design of this study. NM and FM have been involved in drafting the manuscript or revising it critically for important intellectual content. Yl, TM, TU, KT, TK, KK, KH, MK, SS, RU, KT, Kl, HI, and Jl Participated in acquisition and interpretation of data. All authors read and approved the final manuscript.

\section{Acknowledgement}

Part of this study was financially supported by JSPS KAKENHI Grant Number 15 K19836, the Practical Research for Innovative Cancer Control from Japan Agency for Medical Research and development, AMED, and the National Cancer Center Research and Development Fund (26-A-18 and 26-A-28).

\section{Author details}

'Department of Radiation Oncology, National Cancer Center Hospital, 5-1-1 Tsukiji, Chuo-ku, Tokyo 104-0045, Japan. ²Department of Head and Neck Surgery, National Cancer Center Hospital, 5-1-1 Tsukiji, Chuo-ku, Tokyo 104-0045, Japan. ${ }^{3}$ Department of Clinical Laboratory and Pathology, National Cancer Center Hospital, 5-1-1 Tsukiji, Chuo-ku, Tokyo 104-0045, Japan. ${ }^{4}$ Department of Oral Health and Diagnostic Sciences, National Cancer Center Hospital, 5-1-1 Tsukiji, Chuo-ku, Tokyo 104-0045, Japan.

\section{Received: 13 August 2015 Accepted: 29 February 2016}

\section{Published online: 07 March 2016}

\section{References}

1. Cancer Registry and Statistics. Cancer Information Service, National Cancer Center, Japan. http://ganjoho.jp/reg_stat/statistics/dl/index.html\#mortality.

2. Japan Health Promotion \& Fitness Foundation. Ministry of Health, Labour and Welfare. 2008. http://www.health-net.or.jp/tobacco/product/pd100000.html.

3. Kramer S, Gelber RD, Snow JB, Marcial VA, Lowry LD, Davis LW, et al. Combined radiation and surgery in the management of advanced head and neck cancer: Final report of study 73-03 of the Radiation Therapy Oncology Group. Head Neck Surg. 1987;10:19-30.

4. Laramore GE, Scott CB, Al-Sarraf M, Haselow RE, Ervin TJ, Wheeler R, et al. Adjuvant chemotherapy for resectable squamous cell carcinomas of the head and neck: report on Intergroup Study 0034. Int J Radiat Oncol Biol Phys. 1992;23:705-13.

5. Bernier J, Domenge C, Ozsahin M, Matuszewska K, Lefebvre JL, Greiner $\mathrm{RH}$, et al. Postoperative irradiation with or without concomitant chemotherapy for locally advanced head and neck cancer. N Engl J Med. 2004;350:1945-52.

6. Cooper JS, Pajak TF, Forastiere AA, Jacobs J, Campbell BH, Saxman SB, et al. Postoperative concurrent radiotherapy and chemotherapy for high-risk squamous cell carcinoma of the head and neck. N Engl J Med. 2004;350:1937-44.

7. Bernier J, Cooper JS, Pajak TF, van Glabbeke M, Bourhis J, Forastiere A et al. Defining risk levels in locally advanced head and neck cancers: a comparative analysis of concurrent postoperative radiation plus chemotherapy trials of the EORTC (\#22931) and RTOG (\#9501). Head Neck. 2005;27:843-50.

8. Hamoir M, Silver CE, Schmitz S, Takes RP, Rinaldo A, Rodrigo JP, et al. Radical neck dissection: is it still indicated? Eur Arch Otorhinolaryngol. 2013;270:1-4.

9. Ferlito A, Johnson JT, Rinaldo A, Pratt LW, Fagan JJ, Weir N, et al. European surgeons were the first to perform neck dissection. Laryngoscope. 2007;117: 797-802.

10. Schmitz S, Machiels JP, Weynand B, Gregoire V, Hamoir M. Results of selective neck dissection in the primary management of head and neck squamous cell carcinoma. Eur Arch Otorhinolaryngol. 2009;266:437-43.

11. Robbins KT, Medina JE, Wolfe GT, Levine PA, Sessions RB, Pruet CW. Standardizing neck dissection terminology. Official report of the Academy's committee for head and neck surgery and oncology. Arch Otlolaryngol Head Neck Surg. 1991;117:601-5.

12. Robbins KT, Clayman G, Levine PA, Medina J, Sessions R, Shaha A, et al. Neck dissection classification update. Revisions proposed by American Head and Neck Society and the American Academy of Otolaryngology Head and Neck Surgery. Arch Otlolaryngol Head Neck Surg. 2002;128:751-8.

13. Gregoire V, Eisbruch A, Hamoir M, Levendag P. Proposal for the delineation of the nodal CTV in the node positive and the postoperative neck. Radiother Oncol. 2006;79:15-20.

14. Chao KS, Wippold FJ, Ozyigit G, Tran BN, Dempsey JF. Determination and delineation of nodal target volumes for head-and-neck cancer based on patterns of failure in patients receiving definitive and postoperative IMRT. Int J Radiat Oncol Biol Phys. 2002;53:1174-84.

15. Carrillo MM, Martin IT, Lara IM, Almodovar RRJM, Moral DAR. Selective use of postoperative neck radiotherapy in oral cavity and oropharynx cancer: a prospective clinical study. Radiat Oncol. 2013;8:103-10.

16. Bonner JA, Harari PM, Giralt J, Azarnia N, Shin DM, Cohen RB, et al. Radiotherapy plus cetuximab for squamous-cell carcinoma of the head and neck. N Engl J Med. 2006;354:567-78.

17. Murakami N, Yoshimoto S, Matsumoto F, Ueno T, Ito $Y$, Watanabe S, et al. Severe gastrointestinal bleeding in patients with locally advanced head and neck squamous cell carcinoma treated by concurrent radiotherapy and cetuximab. J Cancer Res Clin Oncol. 2015;141:177-84.

18. Eisbruch A, Schwartx M, Rasch C, Vineberg K, Damen E, Van As CJ, et al. Dysphagia and aspiration after chemoradiotherapy for head-and-neck cancer: which anatomic structures are affected and can they be spared by IMRT? Int J Radiat Oncol Biol phys. 2004;60:1425-39.

19. D'Cruz AK, Vaish R, Kapre N, Dandekar M, Gupta S, Hawaldar R, et al. Elective versus therapeutic neck dissection in node-negative oral cancer. N Engl J Med. 2015;373:521-9.

20. Nadiaye C, Mena M, Alemany L, Arbyn M, Castellsaque X, Laporte L, et al. HPV DNA, E6/E7 mRNA, and p16INK4a detection in head and neck cancers: a systematic review and meta-analysis. Lancet Oncol. 2014;15:1319-31.

21. Chung $\mathrm{CH}$, Zhang Q, Kong CS, Harris J, Fertig EJ, Harari PM, et al. p16 protein expression and human papillomavirus status as prognostic biomarkers of nonoropharyngeal head and neck squamous cell carcinoma. J Clin Oncol. 2014;32:3930-8.

22. Grabenbauer G, Mühlfriedel C, Rödel F, Niedobitek G, Hornung J, Rödel C, et al. Squamous cell carcinoma of the oropharynx: Ki-67 and p53 can identify patients at high risk for local recurrence after surgery and postoperative radiotherapy. Int J Radiat Oncol Biol Phys. 2000;48:1041-50.

23. Biau J, Chautard E, Miroir J, Lapeyre M. Radioresistance parameters in head and neck cancers and methods to radiosensitize. Cancer Radiother. 2015. Epub ahead of print. 